\title{
Phenotypes of Lung Mononuclear Phagocytes in HIV Seronegative Tuberculosis Patients: Evidence for New Recruitment and Cell Activation
}

\author{
José R Lapa e Silva*/+, Cristiane Linhares, Neio Boechat, Lorena Rego, Maria \\ da Glória Bonecini Almeida**, Afrânio L Kritski, John L Ho***
}

\begin{abstract}
Laboratório Multidisciplinar de Pesquisa e Serviço de Pneumologia, Hospital Universitário Clementino Fraga
Filho, Universidade Federal do Rio de Janeiro, RJ, Brasil *Laboratório de Anatomia Patológica, Hospital

Evandro Chagas, Av. Brasil 4365, 21045-900, Rio de Janeiro, RJ, Brasil **Laboratório de AIDS e Imunologia Humana, Instituto Oswaldo Cruz, RJ, Brasil ***Division of International Medicine and Infectious Diseases,

Cornell University Medical College, New York, NY, USA
\end{abstract}

Mycobacterium tuberculosis preferentially resides in mononuclear phagocytes. The mechanisms by which mononuclear phagocytes keep M. tuberculosis in check or by which the microbe evades control to cause disease remain poorly understood. As an initial effort to delineate these mechanisms, we examined by immunostaining the phenotype of mononuclear phagocytes obtained from lungs of patients with active tuberculosis. From August 1994 to March 1995, consecutive patients who had an abnormal chest $X$-ray, no demostrable acid-fast bacilli in sputum specimens and required a diagnostic bronchoalveolar lavage (BAL) were enrolled. Of the 39 patients enrolled, 21 had microbiologically diagnosed tuberculosis. Thirteen of the 21 tuberculosis patients were either HIV seronegative $(n=12)$ or had no risk factor for HIV and constituted the tuberculosis group. For comparison, M. tuberculosis negative patients who had BAL samples taken during this time $(n=9)$ or normal healthy volunteers $(n=3)$ served as control group. Compared to the control group, the tuberculosis group had significantly higher proportion of cells expressing markers of young monocytes (UCHM1) and RFD7, a marker for phagocytic cells, and increased expression of HLA-DR, a marker of cell activation. In addition, tuberculosis group had significantly higher proportion of cells expressing dendritic cell marker (RFD1) and epithelioid cell marker (RFD9). These data suggest that despite recruitment of monocytes probably from the peripheral blood and local cell activation, host defense of the resident lung cells is insufficient to control M. tuberculosis.

Key words: pulmonary tuberculosis - alveolar macrophages - activation - monoclonal antibodies

Tuberculosis, caused by Mycobacterium tuberculosis, is the single most important infectious disease of mankind. It is estimated to infect one-third of the world population, and anually causes over 3 million deaths (Raviglione et al. 1995). Inhalation of as few as three bacilli in a droplet nuclei of 5 $\mu \mathrm{m}$ or less can result in lung infection. M. tuberculosis preferentially infects and resides in mononuclear phagocytes. The mechanisms by which mononuclear phagocytes keep $M$. tuberculosis in check or by which the microbe evades control to

This work was supported in part by grants from the Brazilian Ministry of Health (024/94 DST/AIDS), Fundação Universitária José Bonifácio/FUJB, Brazilian Research Council/CNPq, and grants from the National Institutes of Health (USA) (R37-22624, D43-TW00018, and AI33322).

${ }^{+}$Corresponding author. Fax: 55-21-270.2193

Received 7 December 1995

Accepted 10 January 1996 cause disease remain poorly understood (Dannenberg \& Rook 1994). Several lines of evidence suggest that mononuclear phagocytes play an essential role in control of tuberculosis. Mice chronically inoculated with silicate have increased susceptibility to M. tuberculosis (Gros et al. 1983) probably due to impairment of macrophage functions. Moreover, in man, tuberculosis in the setting of silicosis is a more aggressive disease and is associated with increased mortality despite adequate treatment (Paul 1961).

By analogy to animal models, it is thought that recruitment of immune cells from the peripheral circulation and development of cell mediated immunity (CMI) result in activation of macrophages for control of intracellular M. tuberculosis. After the initial infection is established, increased monocytopoiesis and premature release of monocytes from the bone marrow lead to monocytosis and accumulation of recently recruited, immature monocytes at the sites of infection (Schmitt et al. 1977). Development of CMI is contingent on pre- 
sentation of mycobacterial antigens in the context of major histocompatibility antigens interacting with T cells. Mycobacterial antigen specific T cells are thought to produce cytokines, such as interferon- $\gamma$ (Dannenberg \& Tomashefski 1988), that in turn activate macrophages. This activation is associated with increased macrophage expression of HLA-DR in conditions such as sarcoidosis (Campbell et al. 1986). Also, the different stages of maturation and activation are associated with expression of surface antigens on macrophages, such as RFD1 and RFD7 (Spiteri et al. 1992). Newly recruited monocytes can be distinguished from long-lived resident cells by the expression of the marker UCHM1 (Hogg et al. 1984).

Recently, probing of disease sites in the lungs by bronchoalveolar lavage has added a new instrument for investigating the mechanisms of containment or evasion of M. tuberculosis (Ainslie et al. 1992, Kuo \& Yu 1993, Hoheisel et al. 1994), but few studies have concentrated on the role of macrophages. As an initial effort to delineate mechanisms by which the microbe evades control, we sought to characterize the immune cells obtained from the lung of patients with active tuberculosis. In this report, we specifically examined by immunohistochemical methods the phenotypes of lung mononuclear phagocytes.

\section{PATIENTS AND METHODS}

Patient selection and ethical considerations All patients included in the study were recruited in the wards and outpatient clinics of the University Hospital Clementino Fraga Filho (HUCFF), Federal University of Rio de Janeiro. They were adults, with abnormal chest X-ray, clinical history and physical exam suggestive of tuberculosis, without sputum or with sputum negative for acid-fast bacilli, that required fiberoptic bronchoscopy and bronchoalveolar lavage (BAL) for elucidation of the diagnosis and therapeutic guidance. These procedures were indicated on clinical grounds by independent physicians. Exclusion criteria were presence of renal or hepatic failure, diabetes or current use of anti-tuberculosis therapy. Once verified by members of the research team the possible elegibility of the patient for the study, his doctor was approached for authorization, and the patients were informed of the objectives and methods of the study, including the need for HIV testing, and invited to sign the Informed Consent Term. HIV testing was preceeded and followed by counselling. No financial retribution was involved. The protocol was approved by the Human Rights Committee of the HUCFF and Cornell University Medical Center. The materials used for the study were truly excess from those employed for diagnosis. Patients for the control group were selected fol- lowing the same principles among patients performing BAL for diagnosis of lung nodules or tumours. Normal volunteers were recruited among participants of the study.

Once the final diagnosis was reached, the patients were included in two groups:

Group I - Patients with active pulmonary tuberculosis without immunodepression: adults, with tuberculosis confirmed by culture, negative serology for HIV by ELISA and/or Western Blot;

Group II - Controls: adults, with diagnosis of lung cancer $(n=3)$, sequelae of previous tuberculosis $(n=3)$, respiratory infections $(n=3)$ or normal volunteers $(\mathrm{n}=3)$, culture negative for $M$. $t u$ berculosis, negative serology for HIV by ELISA and/or Western Blot.

Procedures - All participants were submitted to a questionnaire, chest X-ray, and cutaneous test with PPD (RT23, Serum Institute, Denmark). BAL was performed following established routine of the Pneumology Unit, complying with international experience. Briefly, following injection of atropin and light sedation, local anaesthesia of upper and lower aiways was performed with lignocaine $2 \%$ and a fiberoptic bronchoscope introduced in the bronchi. Five to six aliquots of $20 \mathrm{ml}$ sterile saline were introduced and aspirated. At least $50 \%$ of the injected fluid was aspirated. After the necessary volume of fluid for diagnostic tests was collected, the remaining fluid was placed in syliconized bottles kept on ice and quickly transported to the laboratory.

Handling of samples - Stringent safety measures were applied throughout the study by all involved staff. Collected volumes were measured, resuspended, a small aliquot taken for cell count in Neubauer chamber and assessment of viability by Trypan blue exclusion. Fluids were filtered through sterile gauze into centrifuge tubes, spun at $4^{\circ} \mathrm{C}$ at $400 \mathrm{~g}$ by $10 \mathrm{~min}$. Supernatant were collected in Eppendorf tubes and frozen for future use. Cell pellets were resuspended in culture medium, washed twice, and finally resuspended to contain $10^{6} \mathrm{cells} / \mathrm{ml}$. Slides were prepared using Cytospin 3 Shandon, with 50.000 cells per well, left to dry under fan, fixed in chloroform/acetone $\mathrm{v} / \mathrm{v}$ for 10 min, wrapped in plastic film and kept at $-20^{\circ} \mathrm{C}$ prior to use.

Immunocytochemistry - The phenotypes of immunocompetent cells obtained by BAL were analyzed by immunocytochemical method using a panel of monoclonal antibodies (Table I). Alkaline phosphatase-anti-alkaline phosphatase (APAAP) was employed. Briefly, cytospins were taken from the freezer $20 \mathrm{~min}$ prior to the staining procedure, a ring of hydrophobic pen (Sigma Co., St. Louis, USA) was traced around the cytospins, and the first layer monoclonal antibody, diluted 
TABLE I

Panel of monoclonal antibodies

\begin{tabular}{lcll}
\hline Name & Isotype & \multicolumn{1}{c}{ Specificity } & \multicolumn{1}{c}{ Reference } \\
\hline RFD1 & IgM & Dendritic cells & Poulter et al. 1986 \\
RFD7 & IgG & Mature macrophages & Poulter et al. 1986 \\
RFD9 & IgG & Epithelioid cells & Munro et al. 1987 \\
RFDR2 & IgG & MHC Class II & Janossy et al. 1986 \\
UCHM1 & IgG & Monocytes & Hogg et al. 1984 \\
\hline
\end{tabular}

Source of the monoclonal antibodies: Royal Free Hospital School of Medicine, London

$\mathrm{MHC}=$ major histocompatibility complex

accordingly in tris-buffered saline (TBS), was applied onto the slides. The slides were incubated for $60 \mathrm{~min}$ in a wet chamber and washed in three changes of TBS, 5 min each. Rabbit immunoglobulin (Ig) against mouse Ig (Z259, Dakopatts a/s, Copenhagen, Denmark) and mouse APAAP (D651, Dakopatts) were employed as described previously (Lapa e Silva et al. 1993a), each step lasting $30 \mathrm{~min}$ and followed by washing in three changes of TBS. Development was performed by incubating the slides with the substrate Fast Red TR (Sigma) and naphtol AS MX phosphate (Sigma) for $15 \mathrm{~min}$, followed by washing in running water and light hematoxylin counterstaining. Positive cells stained in red or dark pink and at least 500 cells per cytospin were counted, the percents of positive cells established in relation to the total number of cells or the total number of macrophages.

Optic densitometry for HLA-DR expression Cytospins were stained as above but were not counterstained by hematoxylin. The stained slides were analyzed using the morphometric software IMASYS (Bioscan Inc., Edmonds, USA) that gives the optic density of the staining, by determining the mean log inverse grey value of a given object in the screen. At least 20 random fields were counted and the mean density determined for each sample (Lapa e Silva et al. 1993b).

Data analysis - The medians plus or minus standard error of the percents of positive cells for each marker were calculated for tuberculosis and control groups and compared by Mann-Whitney test, the results being considered statistically significant when $p<0.05$. For the optic densitometry of HLADR expression, medians plus or minus standard error of optic density were calculated and compared as above.

\section{RESULTS}

From August 1994 to March 1995, 39 patients were enrolled in the study according to entry criteria. Twenty one patients had bacteriologically confirmed tuberculosis. Eight of the 21 were HIV seropositive and were excluded from this analysis. For the current study 13 of the 21 documented tuberculosis patients, called Group I, were studied. For comparison, a control group, called Group II, was studied; they included 3 normal healthy volunteers and 9 patients that had neither tuberculosis nor HIV infection [neoplasia (3); treated, old tuberculosis (3); lung infiltrates that disappeared either spontaneously or after short course of antibiotics (3)]. One patient from each group refused HIV test but lack risk factors for HIV infection. The demographic, clinical and laboratory data from groups I and II are presented in Table II.

TABLE II

Demographic, clinical and laboratory data

\begin{tabular}{lcc}
\hline & Group I $(\mathrm{n}=13)$ & Group II $(\mathrm{n}=12)$ \\
\hline Age & $33.2 \pm 3.0$ & $44.8 \pm 4.0$ \\
Sex & $5 \mathrm{~F}, 8 \mathrm{M}$ & $4 \mathrm{~F}, 9 \mathrm{M}$ \\
Smokers & $4(14 \mathrm{p} / \mathrm{y})$ & $7(13 \mathrm{p} / \mathrm{y})$ \\
Occupational risks & 0 & 2 \\
Associated diseases & 1 & 4 \\
Drugs & 0 & 0 \\
PPD reactor & $10(3$ unk. $)$ & $8(4$ unk. $)$ \\
BCG scar & 5 & 5 \\
HIV+ & 0 & 0 \\
Localized lesion at CXR & 9 & - \\
AFB+ in BALF & 3 & - \\
Good therapy outcome & 12 & - \\
\hline
\end{tabular}

Group I = TB+HIV-; Group II = TB-HIV-; p/y = pack/ years; unk. = unknown; $\mathrm{CRX}=$ chest $\mathrm{X}$-ray; $\mathrm{AFB}=$ acid-fast bacilli; $\mathrm{BALF}=$ bronchoalveolar lavage flluid

All patients were followed in the hospital's outpatient clinic. Tuberculosis therapy consisted of rifampin, isoniazid and pyrazinamide for two months, followed by rifampin and isoniazid for four months. All patients responded to treatment. One patient died from sepsis unrelated to the lung condition during treatment, one month after the start of therapy for tuberculosis.

Bronchoalveolar lavage - Patients from each group had similar volume of fluid recovered and number of total cells, or cell viability. The medians of cells obtained by BAL was $1.1 \pm 0.3 \times 10^{7}$ $\left(1.6 \times 10^{6}-3.1 \times 10^{7}\right)$ in Group I and $1.4 \pm 0.3 \times$ $10^{7}\left(2.0 \times 10^{6}-2.6 \times 10^{7}\right)$ in Group II. Differences between tuberculosis and control groups were seen in neutrophil and eosinophil counts, increased in Group I, with consequent fall in relative proportions of macrophages in tuberculosis in relation to controls (Table III). No differences were found, however, in absolute numbers of macrophages between the two groups (data not shown). 


\section{TABLE III}

Differential cell counts in bronchoalveolar lavage in percents of the total cells

\begin{tabular}{llll}
\hline & $\begin{array}{l}\text { Group I } \\
(\mathrm{n}=13)\end{array}$ & $\begin{array}{l}\text { Group II } \\
(\mathrm{n}=12)\end{array}$ & $\mathrm{p}$ \\
\hline Macrophages & $80.5 \pm 5.2$ & $87.3 \pm 2.0$ & $<0.05$ \\
Lymphocytes & $12.9 \pm 1.3$ & $9.7 \pm 0.8$ & $\mathrm{NS}$ \\
Neutrophils & $1.8 \pm 5.3$ & $0.8 \pm 0.2$ & $<0.05$ \\
Eosinophils & $2.1 \pm 0.2$ & $1.2 \pm 0.2$ & $<0.05$ \\
Epithelial cells & $0.9 \pm 0.3$ & $1.2 \pm 0.4$ & $\mathrm{NS}$ \\
\hline
\end{tabular}

Group I = TB+HIV-; Group II = TB-HIV-; Medians \pm standard error of percents of cell type; $p$ values between Groups I and II by Mann-Whitney test; NS= not significant.

Phenotype of alveolar macrophages - Fig. 1 illustrates the proportions of lung macrophages that were young monocytes, as defined by the marker UCHM1, and macrophages exhibiting phagocytic ability (defined by RFD7). Young monocytes were 6-fold higher in tuberculosis group than in control group. Macrophages with phagocytic ability showed a two-fold increase in tuberculosis group.

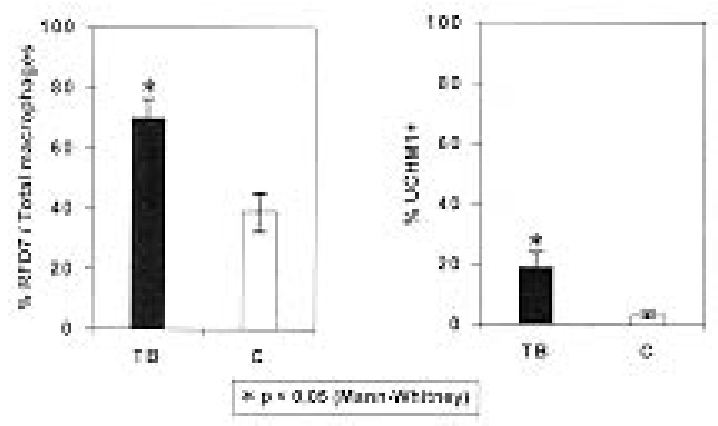

Fig. 1: frequency of mature macrophages, as stained by RFD7, and monocytes, as stained by UCHM1, in tuberculosis (solid bar) and control (open bar) groups. Results represent the medians of percents of positive cells in relation to total number of macrophages \pm standard errors.

We additionally evaluated macrophages expressing dendritic and epithelioid phenotype. As illustrated in Fig. 2, tuberculosis patient group had a 10 -fold increase in epithelioid cells and a 6 -fold increase in the dendritic cell marker.

We next examined by quantitative method expression of HLA-DR (Fig. 3). There was a $70 \%$ increase of expression in alveolar macrophages from tuberculosis patients as compared to control group.

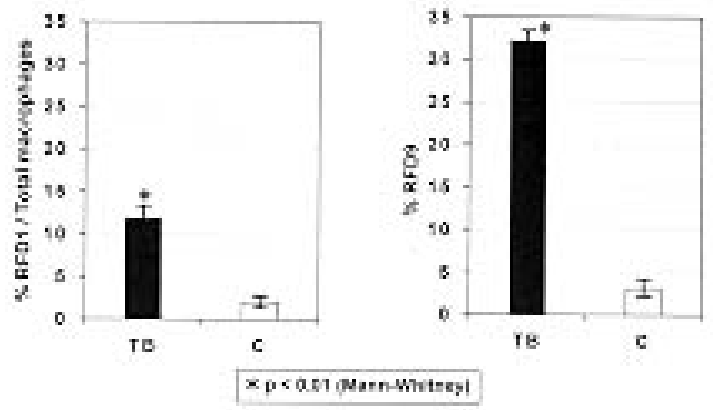

Fig. 2: frequency of dendritic cells, as stained by RFD1, and epithelioid cells, as stained by RFD9, in tuberculosis (solid bar) and control (open bar) groups. Results represent the medians of percents of positive cells in relation to total number of macrophages \pm standard errors.

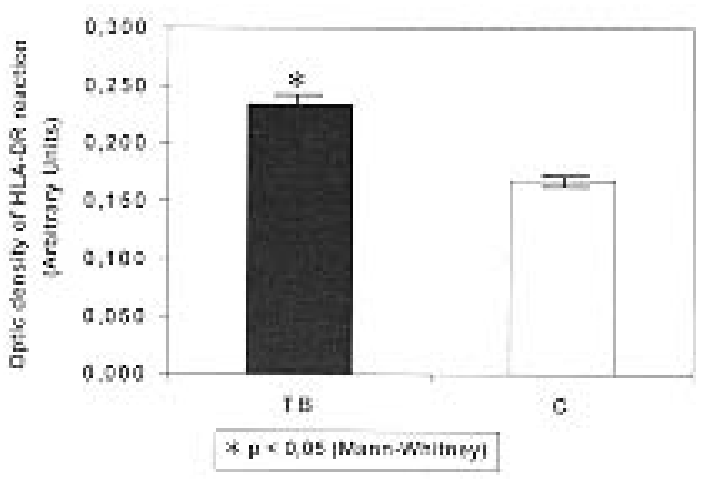

Fig. 3: optic density of HLA-DR reaction, expressed in arbitrary units, in tuberculosis (solid bar) and control (open bar) groups. Results represent the medians of readings \pm standard errors.

\section{DISCUSSION}

Alveolar macrophages are a heterogeneous cell population and the balance between the subpopulations varies according to different disease states (Lohmann-Matthes et al. 1994). Surface markers in macrophages have been evaluated over the last 10 years. However, few studies have concentrated on the phenotype of alveolar macrophages in pulmonary tuberculosis. Kuo and Yu (1993) found heterogeneous distribution of alveolar macrophages obtained by BAL from 12 tuberculosis patients by using Percoll density fractionation. Alveolar macrophages recovered from patients with tuberculosis were mainly in the lower-density fraction, whereas those obtained from normal controls fractionated in the high-density layer. This difference in gradient density distribution was inversely correlated with sputum bacterial load and radiographic disease extent. They also found increased CD4/CD8 ratios in BAL from tuberculosis patients and suggested that this difference may be due to higher activation status of the macrophages from tuberculosis, due to interaction with T lymphocytes. 
The influence of $\mathrm{T}$ lymphocytes on the phenotype of alveolar macrophages was also investigated elsewhere. Striz et al. (1993) found that the phenotypic expression of surface markers such as KiM8, RFD7, RFD9, and others were upmodulated in the presence of lymphocytes from the BAL. Similarly, HLA-DR expression on alveolar macrophages was under influence of BAL lymphocytes and cytokines (Striz et al.1993).

The issue of alveolar macrophage heterogeneity was also investigated in immunodeficiency situations, associated or not with HIV infection. Using a panel of monoclonal antibodies similar to that employed in our study, Bray et al. (1993) studied 30 patients with HIV-related lung conditions and 12 bone marrow recipient individuals undergoing BAL due to pneumonitis. They found a marked decrease in the number of alveolar macrophages stained by CD68, RFD1, RFD7 and a remarkable decrease in the amount of HLA-DR expressed by these cells. They suggested that a defective macrophage population is probably a factor contributing to immunosuppression. Similar findings were reported by Lanza et al. (1993) in 24 patients with HIV-related conditions. They also found a marked decrease in the expression of several antigenic determinants in alveolar macrophages, such as CD68, CD36, CR1, CD11c and HLA-DR, while other markers were mildly affected, such as CD4, CD14, CD45R, CD11b and CD16.

In the present study we found remarkable evidence of upmodulation of all macrophage markers employed, including evidences of macrophage activation. The quantification of HLA-DR expression by optic densitometry demonstrated a significant increase in the amount of this marker in alveolar macrophages obtained from tuberculosis sites. Increased expression of HLA-DR is usually taken to represent cell activation and augmented ability for antigen presentation. This has been previously demonstrated in other inflammatory conditions of the lung, including sarcoidosis (Campbell et al. 1986) and experimental bronchiectasis (Lapa e Silva et al. 1993b) and has been associated with increase amounts of interferon- $\gamma$. Of interest is the additional finding of interferon- $\gamma$ in BAL fluid in patients who had high bacillary load as indicated by positive acid-fast bacilli (data not shown).

Another interesting finding was the highly significant increase, around 10-fold, in the expression of the marker RFD9 in tuberculosis as opposed to controls. In immunohistochemical studies, this marker stains macrophages, including epithelioid cells and giant cells, inside granulomata of different origins, such as sarcoidosis and tuberculoid leprosy (Munro et al. 1987). These cells are highly differentiated, activated cells. However, increased expression of this marker in alveolar macrophages from tuberculosis sites was never demonstrated before, and suggests that alveolar macrophages acquire the marker during the immune response against $M$. tuberculosis, probably in the process of containment of the bacilli that results in granuloma formation.

These findings pose a paradox of macrophage activation and yet inability to erradicate the tubercle bacilli. A potential explanation is our unpublished finding that in BAL samples of these patients we could find the presence of an increased subpopulation of CD4+CD7- T cells. These cells have been found in HIV-infected patients to act as suppressor cells (Autran et al. 1995). Thus, despite exhibiting activation markers, these macrophages may be down-modulated and less able to erradicate the ingested $M$. tuberculosis, resulting in active disease. Alternatively, virulence determinants (not measured in this paper) may account for adequate activation state of macrophages but inability to contain M. tuberculosis.

An additional observation is the finding of increased percentage of mononuclear phagocytes expressing UCHM1, a marker for monocytes. Presumably these monocytes are recently recruited from the blood. Although monocytes compared to macrophages have higher oxidative capacity, producing more reactive oxigen intermediates, the M. tuberculosis expression of catalase (that catalyses the detoxification of $\mathrm{H}_{2} \mathrm{O}_{2}$ to water), makes this defense-effector function ineffective. The newly recruited monocytes have lower antigen-presenting capacity in comparison to macrophages, additionally contributing to lowered cell mediated immunity. They may also not have had adequate time to interact with cells or cytokines in the lung parenchyma that prepare these cells for more effective control of M. tuberculosis. Furthermore, in vitro observation by our laboratory have demonstrated that peripheral blood mononuclear cells (monocytes) are unable to control $M$. tuberculosis growth. In fact, the growth of $M$. tuberculosis within monocytes approximates that in 7H9 Middlebrook enriched medium. Therefore, although recruitment of monocytes is required for the continuation of the immune response against the bacilli, they may also act to amplify clinical disease.

Our findings point out to macrophage activation and recruitment of monocytes into the lung in the setting of acute pulmonary tuberculosis. Future investigations of potential mechanisms previously outlined whereby disease occurs despite macrophage activation may lead to novel strategies for therapeutic intervention. 


\section{ACKNOWLEDGEMENTS}

To Dr LW Poulter and Prof. G Janossy, Academic Department of Clinical Immunology, Royal Free Hospital School of Medicine, London, UK, for the gift of the monoclonal antibodies, to Prof. WD Johnson Jr, Division of International Medicine and Infectious Diseases, Cornell University Medical School, New York, USA, for generous support and advice, to Mrs Cesonia Martinusso for expert technical help, and to the students Alessandra Fonseca, Aline L do Amor Divino, Bianca B Torres, Flávia B Costa e Ivone O Morgado for collaboration during the study.

\section{REFERENCES}

Ainslie GM, Solomon JA, Bateman ED 1992. Lymphocyte and lymphocyte subset number in blood and in bronchoalveolar lavage and pleural fluid in various forms of human pulmonary tuberculosis at presentation and during recovery. Chest 102: 54-59.

Autran B, Legac E, Blanc C, Debré P 1995. A Th0/ Th2-like function of CD4+CD7- T helper cells from normal donors and HIV-infected patients. J Immunol 154: 1408-1417.

Bray DH, Squire SB, Bagdades E, Mulvenna PM, Johnson MA, Poulter LW 1993. Alveolar macrophage populations are distorted in immunocompromised patients with pneumonitis. Eur Respir J 5: 545-552.

Campbell DA, Du Bois RM, Butcher RG, Poulter LW 1986. The density of HLA-DR expression on alveolar macrophages is increased in pulmonary sarcoidosis. Clin exp Immunol 65: 165-171.

Dannenberg AM Jr, Rook GAW 1994. Pathogenesis of pulmonary tuberculosis: an interplay of tissuedamaging and macrophage activating immune responses - dual mechanisms that control bacillary multiplication, p. 32-54. In BR Bloom, Tuberculosis: pathogenesis, protection, and control. American Society for Immunology, Washington, DC.

Dannenberg AM Jr, Tomashefski JF Jr 1988. Pathogenesis of pulmonary tuberculosis, p. 1821-1842. In AP Fishman Pulmonary diseases and disorders. MacGraw-Hill Book Co., NewYork.

Gros P, Skamene E, Forget A 1983. Cellular mechanisms of genetically controlled host resistance to Mycobacterium bovis (BCG). J Immunol 131: 19661973.

Hoheisel GB, Tabak L, Teschler H, Erkan F, Krogel C, Costabel U 1994. Bronchoalveolar cytology and immunocytology in pulmonary tuberculosis. Am J Respir Crit Care Med 149: 460-463.

Hogg N, MacDonald S, Slusarenko M, Beverley PCL
1984. Monoclonal antibodies specific for human monocytes, granulocytes and endothelium. Immunology 53: 753-767.

Janossy G, Bofill M, Poulter LW, Rawlings E, Burford GD, Navarrete C, Ziegler A, Kelemen E 1986. Separate ontogeny of two macrophage-like accessory cell populations in the human fetus. J Immunol 136: 4354-4361.

Kuo HP, Yu CT 1993. Alveolar macrophage subpopulations in patients with active pulmonary tuberculosis. Chest 104: 1773-1778.

Lanza F, Ferrari L, Sighinolfi L, Ghinelli F, Latorraca A, Moretti S, Trevisani L, Potena A, Malavasi F, Castoldi G 1993. Reduced expression of macrophage-associated antigens on alveolar mononuclear phagocytes from acquired immunodeficiency syndrome. Int J Clin Lab Res 23: 146-150.

Lapa e Silva JR, Bachelet CM, Pretolani M, Baker D, Scheper RJ, Vargaftig BB 1993a. Immunopathological alterations in the bronchi of immunized guinea pigs. Am J Respir Cell Mol Biol 9: 44-53.

Lapa e Silva JR, Guerreiro D, Munro NC, Poulter LW, Cole PJ 1993b. Immunopathology of chronic bronchial inflammation: Contribution of animal models, p. 266-279. In JP Tarayre, B Vargaftig, E Carilla (eds). New Concepts in Asthma. MacMillan Press, London.

Lohmann-Matthes ML, Steinmüller C, Franke-Ulmann G 1994. Pulmonary macrophages. Eur Respir J 7: 1678-1689.

Munro CS, Campbell DA, Collings LA, Poulter LW 1987. Monoclonal antibodies distinguish macrophages and epithelioid cells in sarcoidosis and leprosy. Clin exp Immunol 68: 282-287.

Paul R 1961. Silicosis in Northern Rhodesia copper mines. Arch Environ Health 2: 96-107.

Poulter LW, Campbell DA, Munro C, Janossy G 1986. Discrimination of human macrophages and dendritic cells by means of monoclonal antibodies. Scand $J$ Immunol 24: 351-357.

Raviglione MC, Snider DE, Kochi A 1995. Global epidemiology of tuberculosis. JAMA 273: 220-226.

Schmitt E, Meuret G, Stix L 1977. Monocyte recruitment in tuberculosis and sarcoidosis. Br J Haematol 35: 11-17.

Spiteri MA, Clarke SW, Poulter LW 1992. Alveolar macrophages that suppress $\mathrm{T}$-cell responses may be crucial to the pathogenetic outcome of pulmonary sarcoidosis. Eur Respir J 5: 394-403.

Striz I, Wang YM, Svarkova I, Trnka L, Sorg C, Costabel U 1993. The phenotype of alveolar macrophages and its correlation with immune cells in bronchoalveolar lavage. Eur Respir J 6: 1287-1294. 